Volume 8. No. 9, September 2020

International Journal of Emerging Trends in Engineering Research

Available Online at http://www.warse.org/IJETER/static/pdf/file/ijeter200892020.pdf

https://doi.org/10.30534/ijeter/2020/200892020

\title{
Construction of an Aircraft-Type UAV for Flight Along a Given Trajectory in the Automatic Mode
}

\author{
Serhii Lienkov ${ }^{1}$, AlexanderMyasischev ${ }^{2}$, Oksana Banzak $^{3}$, Larysa Komarova ${ }^{4}$, Nataliia Lytvynenko ${ }^{5}$, \\ Oleg Miroshnichenko ${ }^{6}$ \\ ${ }^{1}$ Research Center, Military Institute of Taras Shevchenko National University of Kyiv, \\ Kyiv, Ukraine, lenkov_s@ukr.net \\ ${ }^{2}$ Department of Telecommunications and Radio Engineering, Khmelnitsky National University, \\ Khmelnitsky, Ukraine, alex56ma@gmail.com \\ ${ }^{3}$ Odessa State Academy of Technical Regulation and Qualityv, Odesa, Ukraine, banzakoksana@ gmail.com \\ ${ }^{4}$ Odessa National Academy of Telecommunications, Kyiv, Ukraine, lacosta_k@ukr.net \\ ${ }^{5}$ Research Center, Military Institute of Taras Shevchenko National University of Kyiv, \\ Kyiv, Ukraine,n123n@ukr.net \\ ${ }^{6}$ Research Center, Military Institute of Taras Shevchenko National University of Kyiv, \\ Kyiv, Ukraine, aleg395@meta.ua
}

\begin{abstract}
A budget (up to $\$ 100$ ) unmanned aerial vehicle (UAV) of an aircraft type based on the OMNIBUSF4V3 flight controller with an STM32F405 microcontroller is developed in the article. The created aircraft performs the following flight modes: the holding altitude and position, the automatic return to the takeoff point on command from the control panel or in case of loss of communication with it, the automatic flight along a given trajectory. The possibility of the flying along the points of the designed aircraft with the firmware INAV ver.2.5.0 without using a barometer and magnetometer, that are necessary for the rotary UAV to perform a similar task, is shown. Using the Blackbox INAV 2.5.0 toolkit and the Google Earth Pro service, the real flight path of the aircraft, speed parameters, and flight altitude according to the readings of the GPS receiver were established. The heights and speeds of the aircraft passing the flight points have been experimentally determined. Their close correspondence was established to those values, that were programmatically entered into the aircraft flight controller before launch. The correspondence was established between the real flight path of the aircraft and the one entered into the flight controller using the configurator.

The constructed aircraft can be used to perform photo and video surveys of the terrain in automatic mode with the route length of 6-8 km, using a lithium polymer battery with a capacity of 1800-2200 mAh.
\end{abstract}

Key words: OMNIBUSF4V3, INAV 2.5, GPS receiver, STM32F405, UAV, GY-NEO6MV, ESC controller, Google Earth Pro, MPU6000, Firefly q6, FlySky FS-i6, Failsafe.

\section{INTRODUCTION}

Currently, it is of interest to build vehicles, that fly along a given trajectory in a fully automatic mode [1-5]. They can be used for terrain research, rescue operations, in the work of fire services, military intelligence. Of particular interest are the vehicles with a fixed wing (the flying wing, the airplane) [2], that are more energetically economical compared to the quadrocopters and hexacopters [6,7], they fly over a greater distance with a higher speed. Therefore, with the same battery capacity and flight weight, they can explore an area 2-3 times larger in size, than the rotary-type UAV (quadcopter). In this regard, the attention is paid to the creation and experimental study of the fixed-wing UAVs (aircraft). The apparatus, considered in the article, is assembled from fairly common budget components, uses free software products, that are open to correction and supports the following flight modes [8]:

1. The horizon holding. A gyroscope and an accelerometer are used.

2. A given height maintaining. The GPS receiver requires. Unlike rotor-type UAVs, the barometer isn't used, that simplifies and reduces the cost of the design.

3. A position maintaining both in the horizontal plane and in the height. The GPS receiver is being used.

4. The mode of returning to the starting point on command from the control panel and in case of communication loss with the control equipment. Unlike rotary UAVs, that require a magnetometer, barometer and GPS receiver, only the GPS is used here.

5. The flight mode along the trajectory specified on the map. Only the GPS receiver is used.

An STM32F405 microcontroller combined on one board with the gyroscope and the accelerometer, the external GPS 
receiver, as well as software for the microcontroller and the external computer are used for the flight of the aircraft-type $\mathrm{UAV}$, for performing the flight modes presented above. The INAV 2.5 [9] configurator is installed on the computer to change the microcontroller firmware parameters. The firmware is software for the microcontroller that uses mathematical models such as PID controllers [7,10], Kalman filter, complementary filter, dynamic Notch filter, Matrix Filter [11], etc. To ensure stable UAV flight, When the specified flight modes are fulfilled, the firmware is adjusted by selecting parameters that depend on the geometry of the aircraft, the installed propulsion system, sensors, speed parameters, and flight trajectory $[7,10]$. In some cases, the program code is also partially corrected. The aim of the work is to create a budget (no more than \$100) aircraft-type UAV and an experimental study of its capabilities based on the STM32F405 microcontroller and the latest version of the INAV 2.5.0 firmware $[12,13]$. The method for solving the problem is the design and improvement of flight controllers for an aircraft-type UAV, which provides a fully automatic flight along a given route with its video filming on a recording camera, as well as setting up free firmware for performing the above flight modes as a result of numerous flight test tests.

\section{METHODOLOGY}

The solution to this problem consists in designing a flight controller for an aircraft, adjusting the firmware parameters together with performing test flight tests to adjust these parameters and, based on the data obtained from blackbox [12], verifying that the actual flight path is set by the program. A simple standard layout of an aircraft with a rectangular wing, a span of $1000 \mathrm{~mm}$ and a fuselage length of $800 \mathrm{~mm}$ was chosen as the load-bearing part (Fig. 1). The optimization of geometric parameters in order to obtain high aerodynamic parameters of the aircraft wasn't considered in the article.

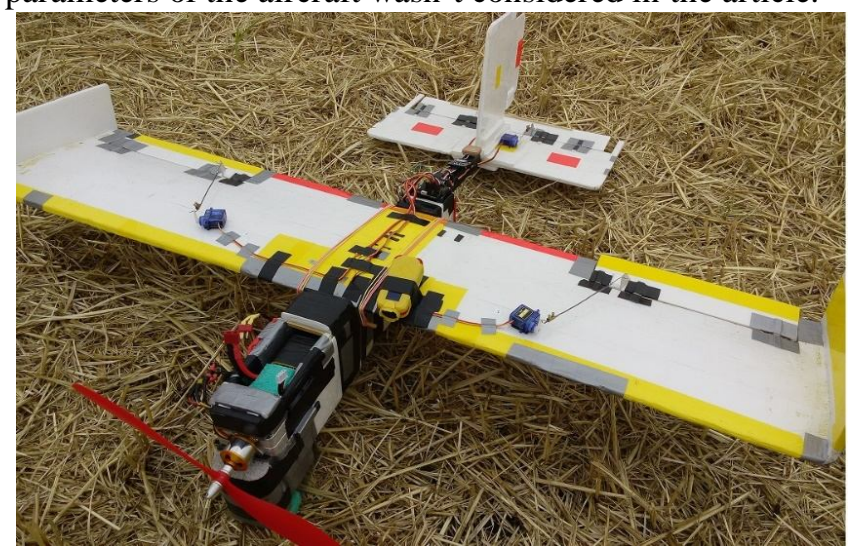

Figure 1: The photo of the tested aircraft

The equipment is installed on the plane:

The motor A2212 / 13T $1000 \mathrm{KV}$ with ESC regulator $30 \mathrm{~A}$; the propeller $12 \times 4.5$ inches; the GPS receiver - module GY-NEO6MV2 - GPS receiver u-blox NEO-6M; the budget control equipment - FlySky FS-i6 flashed from 6 to 10 channels with communication range of up to $1.5 \mathrm{~km}$ [14]; the flight controller - OMNIBUSF4V3 [15] based on STM32F405 LQFP64 microcontroller (168 Mhz, 1M Flash, 192 KB SRAM) with built-in gyroscope, the MPU6000 accelerometer and the BMP280 barometer (not used); battery $1800 \mathrm{mAh}, 11.1 \mathrm{~V}$; the recording camcorder - Firefly q6.

The flight time with such battery was at least $10 \mathrm{~min}$. It's enough for exploring a route with a length of at least $6 \mathrm{~km}$ with a speed set by the program of $36 \mathrm{~km} / \mathrm{h}$.

The connection diagram of the electronic components is shown in Fig. 2. The servos of the wing ailerons, elevators and rudders are connected to a separate $5 \mathrm{~V}$ power supply. The flight controller, the control receiver, the GPS receiver are connected to another $5 \mathrm{~V}$ power supply integrated with the ESC motor controller.

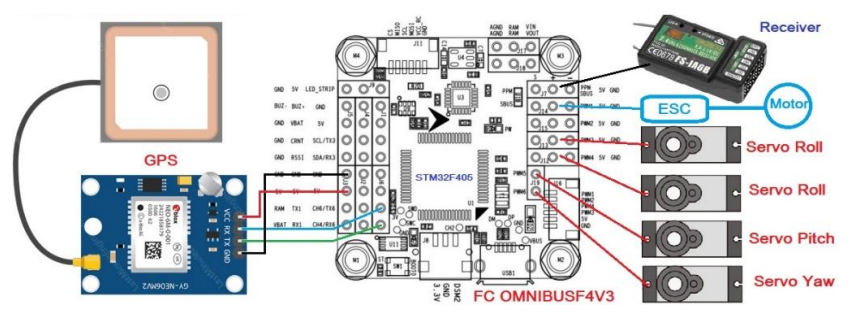

Figure 2: The connection diagram of the main electronic components

The firmware is downloaded to the flight controller from the site: https://github.com/iNavFlight/inav/releases/tag/2.5.0, using the INAV 2.5.0 configurator, that is installed on the computer from the site: https://github.com/iNavFlight/ inav-configurator / releases / tag / 2.5.0. The firmware can also be loaded into the controller, as presented in [16], if there are difficulties in downloading through the configurator.

After starting the INAV 2.5.0 configurator, the computer is connected to the flight controller and the firmware parameters are set depending on the aircraft configuration, equipment used, flight parameters, flight modes. For this, a sequential entry into the tabs of the configurator is performed with the setting of the necessary parameters $[10,16]$. The most necessary settings are discussed below.

The Mixer tab (Fig. 3). The type of the aircraft, the connection diagram of the motors and servos to the flight controller are established. Sets the servo functions, for example, what drives control the elevators (Pitch), directions (Yaw), aileron (Roll). In the Calibration tab, the accelerometer is calibrated according to the scheme, presented in this tab. In the Outputs tab, the protocols for the operation of the ESC motor controller, servo drives are set. The motor and the servos are turned on - the Enable motor and servo output parameter - and the motor is turned off, when the throttle is low - the Stop motors on low throttle parameter. In the Ports tab on the 6th port, the GPS receiver with a speed of $38400 \mathrm{Bit} / \mathrm{s}$ is installed in accordance with the diagram of its connection to the flight controller in Fig. 2. 


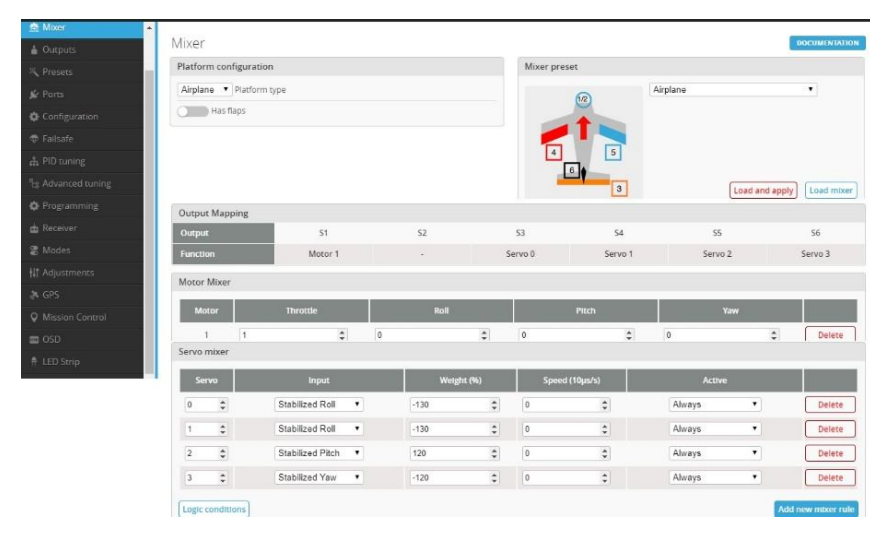

Figure 3: The Mixer tab

In the Configuration tab, the parameters are set, that determine the accelerometer used, the protocol of the GPS receiver, and the takeoff assistant mode is also enabled Permanently Enable Launch Mode for Fixed Wing.

The Failsafe mode is being configured. To do this, use the Failsafe tab and the commands corresponding to this mode, that are entered in the CLI tab.

Below there are the conditions of the INAV firmware, under that failsafe is activated:

1. Any flight channel (pitch, roll, throttle or yaw) doesn't send pulses from the control panel.

2. Any channel is out of range, that can be determined using the commands in the cli tab: "get rx_min_usec; get rx_max_usec". In operation, in the CLI tab, the command "set rx_min_usec $=940$ " is executed. When the control equipment is on, the minimum gas corresponds to a value greater than 1000. When it is off - 900 (this is how the receiver is configured). The value 900 is less than the minimum value of 940. This will trigger Failsafe.

3. FAILSAFE mode is activated with a switch on the remote control.

Before setting up Failsafe, the Flysky FS-iA6 receiver is pre-configured so that when the remote control is turned off, it sends a $900 \mu$ s long pulse to the flight controller via the throttle channel (3rd channel - the throttle), as presented in the source [17]. This means that failsafe will be triggered by condition 2.

In Failsafe, the available commands are executed when a failsafe occurs:

DROP: Turn off the motors and execute disarm (the UAV will fall and be damaged. Usually a parachute is used here).

SET-THR (Land): Assumes a gradual descent. Here you can adjust the roll / pitch / yaw angle when the aircraft is descending, set the engine speed when descending, and set the time after which the engine turns off. The parameters are set experimentally. If the altitude is low and the plane lands in less than 20 seconds (default), the motor and ESCs may fail. A gyroscope and an accelerometer are used.

RTH: (Return to the starting point). The INAV firmware automatically moves the UAV back to its original position and lands or circles within a radius of $50 \mathrm{~m}$ over the landing site for the aircraft. The GPS receiver is used for this.

NONE: Do nothing. It is intended for fully automatic waypoint flight outside radio diode access from the control panel. If the flight path of the aircraft exceeds the range of the control equipment, then this failsafe mode is set.

In the PID tuning tab, the PID parameters of the aircraft regulator are set (Fig. 4). Their determination was carried out by sequential selection during test flights in order to ensure maximum stability of the aircraft. INAV firmware allows automatic tuning according to a special algorithm during manual flight. However, it doesn't always provide the best PID tuning for the controller.

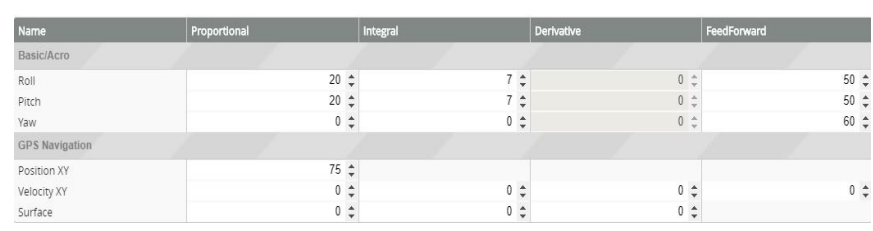

Figure 4: The PID controller parameters

The aircraft flight parameters are set in the Advanced tuning tab. The flight mode is set to Cruise. In this case, the flight controller constantly reads the coordinates from the GPS receiver. The point flight speed is set to $10 \mathrm{~m} / \mathrm{s}$ - the max CRUISE speed parameter $=1000$. When approaching the launch point, the aircraft will describe circles with the radius of $50 \mathrm{~m}$ without landing - the Loiter radius parameter $=5000$. For example, the value of the parameters Cruise throttle and Max. throttle. If they are too small, the altitude hold mode will not be possible. With the insufficiently powerful motor, their values are chosen large. For the aircraft being tested, the Cruise throttle $=1700$ (it's the throttle value for maintaining straight flight while maintaining altitude) and Max. throttle = 1950 - corresponds to the maximum engine speed for taking the aircraft to the set altitude. In this tab, the roll, ascent and dive angles for automatic flight are set. They are chosen depending on the power of the engine and the strength of the aircraft. For the considered aircraft, they have values: 20, 20, 15 degrees, respectively. At least [10] is selected as the mode for returning to the starting point. It returns the plane to the starting point at the altitude not less, than that specified in the RTH altitude parameter (set to $30 \mathrm{~m}$ ). If the aircraft's altitude was less, than the RTH altitude, when the RTH was triggered, then it rises to the return altitude. If more, then it returns at the same height. In this tab, it's possible to set the number of the satellites to that the GPS receiver must be connected in order to be able to confidently fly along the trajectory and to perform the Arming procedure. Six satellites have been used in the work. During testing, the installed GPS receiver in the open area is connected to 9-11 satellites. It was found, that the height determined by him for 15 minutes fluctuated in the range of no more, than $1 \mathrm{~m}$ (at the launch site 323-324 m). Therefore, when configuring the OMNIBUSF4V3 flight 
controller, the barometer installed in it was disabled. As experience with quadrocopters has shown, at speeds of about $30 \mathrm{~km} / \mathrm{h}$, the effect of the barometer "blowing out" occurs, that leads to the incorrect altitude determination.

The Receiver tab sets the protocol used by the receiver PPM and checks the correct operation of the control equipment channels and the throttle channel triggering, when the equipment is powered off to switch to the Failsafe mode.

The airplane flight modes are set on the control panel switches in the Modes tab (Fig. 5).

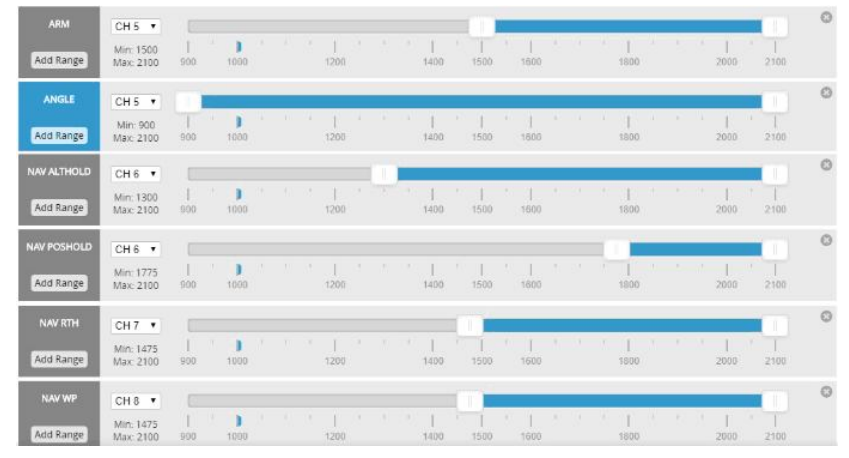

Figure 5: The setting flight modes on the control panel switches

Below is the description of the modes:

ANGLE - provides automatic holding of the aircraft in the horizontal plane using the accelerometer;

NAV ALTHOLD - when you turn on the switch associated with the 6th channel, the flight is performed at the constant altitude determined by the GPS receiver at the time of switching.

NAV POSHOLD - the automatic holding of the horizontal position. If the NAV ALTHOLD mode is on, the position will also be held in altitude. Therefore, the modes NAV ALTHOLD, NAV POSHOLD are linked to the three-position switch. The aircraft maintains the position by flying in the circle with the radius of $50 \mathrm{~m}$ at the constant altitude. The gyroscope, accelerometer, GPS receiver are used;

NAV RTH -the turning on the 7th channel switch results in the aircraft returning to the starting point. When approaching the launch point, the aircraft describes the circle with the radius of $50 \mathrm{~m}$ at the altitude specified by the "At least" and "RTH altitude" parameters of the Advanced tuning tab. The landing is performed in manual mode (ANGLE).

NAV WP - performs the automatic flight to specified points on the geographic map. The launch is performed in manual mode, then this switch is turned on to follow the route. When approaching the launch point, the aircraft, as in the NAV RTH mode, flies in the circle with the radius of 50 $\mathrm{m}$. The landing is carried out in manual mode after turning off the switch of the 8th channel.

In the Mission Control tab (Fig. 6), the aircraft flight is configured along the given trajectory. The computer with the
INAV configurator must be connected to the Internet. The section of the map, where the flight is planned is selected. The waypoints are set by pressing the mouse button. Each waypoint after the second click on it with the mouse displays its coordinates with the parameters of the flight height above it and the speed. These values can be edited. If you need to return to the starting point, check the box on the RTH at the end of the mission parameter. Above it, the plane will circle with the radius of $50 \mathrm{~m}$, that is specified in the Loiter radius parameter in the Advanced tuning tab. The generated route is recorded by the Save mission to FC and Save Eeprom mission commands. The waypoint flight can be performed, if the radio control switch is set to NAV WP flight mode in the Modes tab.
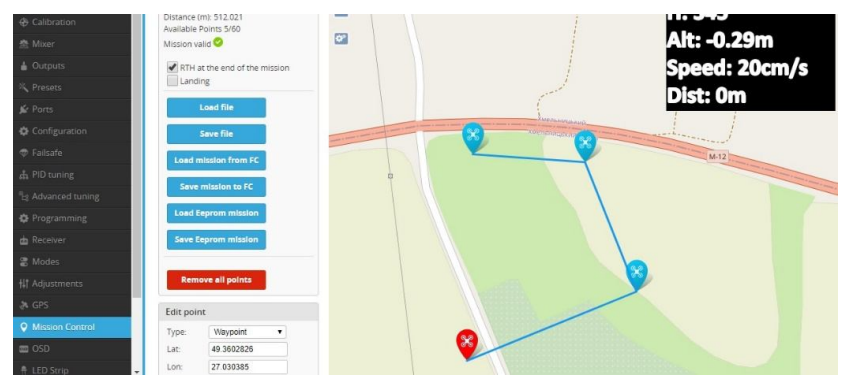

Figure 6: The aircraft flight trajectory formation

The point, to that the plane returns after completing the mission, isn't displayed on the map. This point corresponds to the launch point, that can be anywhere. The sequence of the points flown by the airplane corresponds to the sequence of the forming points on the map.

It is important to note the following feature of the firmware. When the flight controller is disconnected from the battery, the mission is lost. If, after switching on, the mission isn't loaded from the EEPROM memory into the flight controller, then the aircraft will not perform automatic flight along the trajectory after setting the switch on the control panel to the NAV WP mode. Therefore, after connecting the battery on the control panel, it's necessary to move the right stick down, and the left stick to the upper right corner and wait for the buzzer signal. This procedure will load the flight mission and only after that you can the Arming of the aircraft and take off with the subsequent switching of the flight mode to the NAV WP position.

After loading the mission, the Arming will be executed only, if the distance from the plane's launch point to the first waypoint is less than specified in the nav_wp_safe_distance = 10000 parameter ( $100 \mathrm{~m}$ by default), the value of that can be increased to $650 \mathrm{~m}$.

The parameter nav_wp_radius $=100 \mathrm{~cm}$ by default determines the distance of the UAV to the given waypoint in order to accept it as reached. It is for the quadcopter. For the airplane, this parameter must be increased, because it's unlikely, that the airplane will hit the waypoint with the accuracy of $100 \mathrm{~cm}$. Usually $1000-3000 \mathrm{~cm}$ are set for the 
airplane. It is due to the slowness of the GPS receiver (it receives coordinates 5 times per second), the high speed of the airplane and the probable deviation from the trajectory. In operation, in the CLI tab, the command set nav_wp_radius = $1500(15 \mathrm{~m})$ was executed. For example, if the plane's speed is $1000 \mathrm{~cm} / \mathrm{s}$, then it will presumably be in the area of the waypoint for 3 seconds, provided that it follows the route accurately. The GPS receiver will be able to receive the aircraft coordinates 15 times during this time. It is sufficient for the flight controller to execute the reroute command. If the plane, for example, due to the wind deviates from the route and doesn't hit the point with the diameter of $30 \mathrm{~m}$, it will describe the arc and return to re-pass the point until it hits it. The Blackbox tab is used to record parameters on the MicroSD card during the flight of the aircraft, in particular, the trajectory, speed, altitude, etc. (Black box, Fig. 7). The recording is performed automatically immediately after switching to the Arming. The Portion of "flight loop iterations to $\log$ (logging rate)" parameter determines the frequency of the recording to the LOG file. For example, 1/2 $(50 \%)$ means, that the data is written once every two processor cycles. The obtained records in the research were used to determine the correspondence of the real trajectory, speed, flight altitude with that set in the flight controller. The real trajectory, speed, distance traveled is recorded during the flight on the MicroSD according to the indications of the GPS receiver.

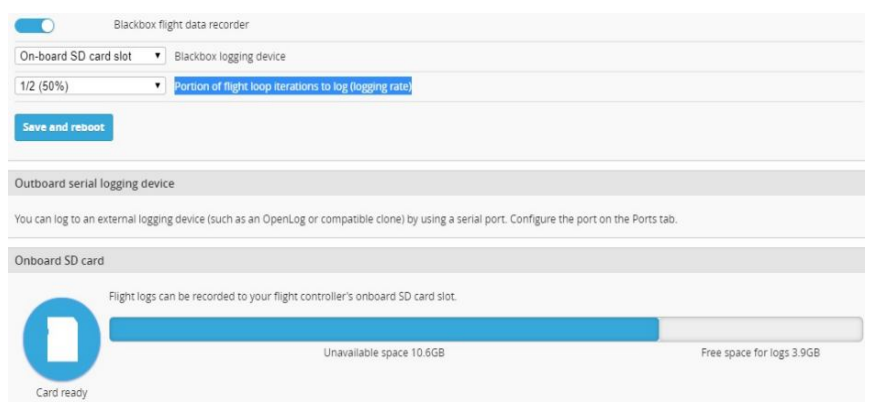

Figure 7: The configuring recording of the flight parameters in the Blackbox tab

In the source [18], you can find the flight recording from the Firefly q6 camera, installed on the wing of the aircraft. The length of the route is about $650 \mathrm{~m}$ with the take-off point. The passage along the route is demonstrated 2 times. At the end of the route, the plane makes circles over the take-off point with the radius of $50 \mathrm{~m}$. The first 3 pictures are the airplane, waypoints on the configurator map, the satellite image (google maps). Further takeoff in manual mode, the flight to points in automatic mode and landing in manual mode.

Let's consider the construction of the real flight path of the aircraft, speed, passing control points, length of the traversed path, flight altitude in the area of the control point. To do this, we will use LOG files created by the flight controller on the
MicroSD card according to the readings of the GPS receiver. The trajectory, speed, altitude viewing was performed, using the Google Earth Pro service [19]. However, to create an airplane flight track with this program, it's necessary to convert the LOG00009.The TXT file from MicroSD to the LOG00009.01.gps.gpx file. We will use the toolkit located at "https://github.com/cleanflight/blackbox-tools/releases/tag/v 0.4.3". Copy the file "blackbox-tools-0.4.3-windows.zip" from there and unpack it. The file "blackbox_decode.exe", appears in the created directory, that is used in the research to convert LOG00009.TXT to LOG00009.01.gps.gpx.

The Fig. 8 shows the flight paths of the aircraft in accordance with the LOG00009.TXT file and the control points inserted there by coordinates from the Mission Control tab (Fig. 6). The first figure on the left is the first pass through the control points, the second figure is two passes and the last figure is the full flight path, that was recorded on the video camera and presented in the source [18]. "Home" is the take-off point, the arrow with the inscription "Blackbox flight log" is the landing point.

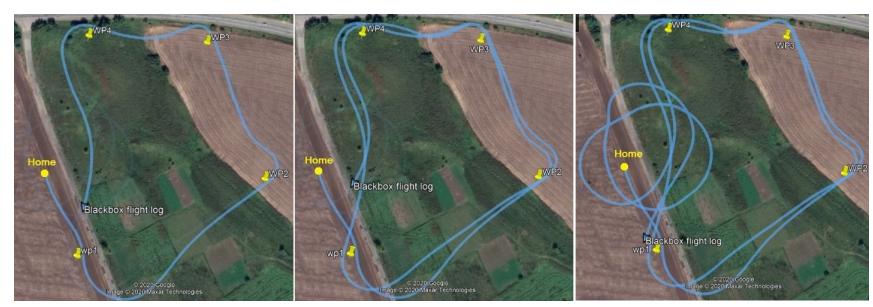

Figure 8: The real plane flight path formed by Google Earth Pro and Blackbox

When you select the "Show terrain profile" parameter in Google Earth Pro, moving the mouse along the lower diagram (below Fig. 9), you can get the altitude, speed, tilt angles of the aircraft along the flight path and compare them with those set in the firmware, using the configurator.

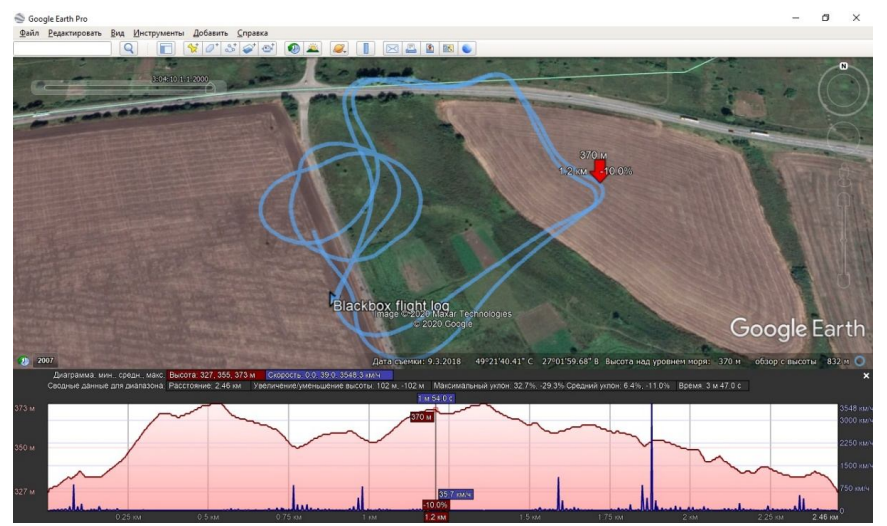

Figure 9: The flight trajectory and the telemetry 
Serhii Lienkov et al., International Journal of Emerging Trends in Engineering Research, 8(9), September 2020, 6145 - 6150

\section{CONCLUSION}

The research results are:

1. The creation of the budget (no more than $\$ 100$ ) UAV of the aircraft type, that is able to perform the following flight modes: the holding altitude and position, the automatic return to the takeoff point on command from the control panel or loss of communication with it, the automatic flight along the given trajectory.

2. The experimentally established possibility of flying along the points of the designed aircraft with the flight controller, based on the STM32F405 microcontroller and firmware INAV ver.2.5.0 without using the barometer and magnetometer, that are necessary for the rotary UAV to perform a similar task.

3. The establishment of the aircraft real flight path, speed parameters, flight altitude according to the readings of the GPS receiver, using the Blackbox INAV 2.5.0 toolkit and the Google Earth Pro service.

4. The determination of the correspondence between the real flight path of the aircraft and entered into the flight controller, using the configurator.

5. The experimentally found correspondence of the altitude and speed of the aircraft passing through the flight points to the values, that were programmatically entered into the aircraft flight controller prior to launch, using the configurator.

\section{REFERENCES}

1. A. Boyko. Fields of application of drones, available at: http://robotrends.ru/robopedia/oblasti-primeneniya-besp ilotnikov.

2. Modernized Spectator Drone from OJS S.P. Korolyova "Meridian Company", available at: https://www.youtube.com/watch?time_continue $=6 \& v=H$ vLErmgBRX4\&feature=emb_logo.

3. N. Lytvynenko, O. Myasischev, S. Lienkov, Yu. Husak, I. Starynskiy. Designing of the Aero Video Intelligence on the STM32H Microcontrollers Basis. International Journal of Innovative Technology and Exploring Engineering, IJITEE. Vol. 9, Is. 8, ISSN: 2278-3075, pp. 88-93, 2020, DOI: 10.35940/ijitee.H6176.069820.

4. S. A. Shvorov, N. A. Pasichnyk, S. D. Kuznichenko, I. V. Tolok., S. V. Lienkov, L. A. Komarova. Using UAV during Planned Harvesting by Unmanned Combines, IEEE 5th International Conference Actual Problems of Unmanned Aerial Vehicles Developments, ISBN: 978-172812592-3, pp. 252-257, 2019.

5. S. Lienkov, G. Zhyrov, O. Sieliukov, I. V. Tolok, A.-S. M. Talib, I. V. Pampukha. Calculation of Reliability Indicators of Unmanned Aerial Vehicle Class ' $\mu$ ' taking into account Operating Conditions at the Design Stagempukha, IEEE 5th International Conference Actual Problems of Unmanned Aerial
Vehicles Developments, ISBN: 978-172812592-3, pp. 52-56, 2019.

6. S. Lienkov, A. Myasischev, O. Banzak, Y. Husak, I. Starynski. Use of rescue mode for UAV on the basis of STM32 microcontrollers. International Journal of Advanced Trends in Computer Science and Engineering. Vol. 9, No.3, ISSN 2278-3091, pp. 3506-3513, https://doi.org/10.30534/ijatcse/2020/156932020.

7. S. Lienkov, A. Myasischev, L. Komarova, N. Lytvynenko, V. Shvab, O. Lytvynenko. Creation of a Rotor-Type UAV with Flight Controllers, Based On a ATmega2560 and STM32f405 Microprocessors. International Journal of Emerging Trends in Engineering Research. Vol. 8, No. 8, ISSN 2347 - 3983, https://doi.org/10.30534/ijeter/2020/104882020.

8. Modes, available at: https://github.com/iNavFlight/inav/wiki/Modes.

9. INAV Configurator 2.5.0, https://github.com/iNavFlight/inav-configurator/release $\mathrm{s} / \mathrm{tag} / 2.5 .0$.

10. A. A. Myasishchev. Features of Implement of INAV Firmware On Omnibusf4v3 Flight Controller for Rotor Type UAV, Khmelnytskyi: ХHУ, № 2, pp. 126-134, 2020.

11. INAV \& EmuFlight Matrix Filter - how does it work? available at:

https://www.youtube.com/watch?v=w0QsVIXYWCE.

12. The Basics of Getting iNav Working on an Airplane, available at:

https://github.com/iNavFlight/inav/wiki/Fixed-wing-gui de.

13. INAV 2.5.0, available at: https://github.com/iNavFlight/inav/releases/tag/2.5.0.

14. FlySky-i6-Mod-10ch, available at: https://github.com/benb0jangles/FlySky-i6-Mod-.

15. OMNIBUS F4V3, available at: http://nic.vajn.icu/PDF/radio-controlled/OMNIBUS_F4 _V3.pdf.

16. A. A. Myasishchev. Possibilities of the CC3D Flight Controller with INAV Firmware, Visnik KhN, Technical sciences, №1, pp. 129-136, 2019.

17. Failsafe quick setup for AWM with Flysky-i6 Setup arducopter failsafe, available at: https://www.youtube.com/watch?v=aZ1A5rAK0uo\&t=1 $27 \mathrm{~s}$.

18. INAV 2.5 by Plane. Waypoint Flight, available at: https://www.youtube.com/watch?v=qRJ8w_ju2YY.

19. Google Earth, available at: https://en.wikipedia.org/wiki/Google_Earth. 